\title{
Preparation and Characterization of Large Size Bulk Nanocrystalline Ni*
}

\author{
Ma Guoliang, Wang Tianqi, Zhang Yanqing, Qi Chunhua, Piao Yinghun, Ma Jianning, Huo \\ Mingxue*
}

Research Center of Basic Space Science: Harbin institute of Technology, Harbin 150001, China

Keywords: electrochemical deposition; nanocrystalline Ni; density; hardness

\begin{abstract}
This work fabricated high quality Nano-Ni using electrochemical deposition through purifying plating solution, adjusting current density and additive. The mean three-dimensional size of nanocrystalline Ni prepared by electrodeposition is larger than $5 \mathrm{~mm}$, the average grain size is $26 \mathrm{~nm}$, the density is $8.8343 \mathrm{~g} / \mathrm{cm}^{3}$, and the average hardness is $384.1 \mathrm{HV}_{1.0}$.
\end{abstract}

\section{Introduction}

In 1960s, Embury, Fischer and Armstrong began to research on ultra-fine grain size materials ${ }^{[1,2]}$. In 1981, Gleiter et al. of the University of Saarland, Germany used inert gas condensation and in-situ pressurization to synthesize nano-pure metals, and set off a research boom in nanomaterials in the international material science world with a landmark paper ${ }^{[3]}$. In July 1990, the first International Conference on nanoscience and technology was held in the United States, marking the birth of nanoscience as a relatively independent discipline ${ }^{[4]}$. When the particle size is reduced to the nanoscale, the periodic boundary conditions of the crystalline material are destroyed and the quasicontinuous energy band is converted into discrete energy levels; The average free path of the electron will be very short, and the coherence and locality will be correspondingly enhanced. This makes nanomaterials with unique surface effect, small size effect, quantum size effect and macroscopic tunneling effect. Therefore, nanomaterials exhibit many excellent properties in mechanical, acoustic, electrical, magnetic, thermal and optical aspects, which are different from those of traditional coarse grain materials ${ }^{[9,10]}$.

The nanocrystalline metal and alloy can be used as the active component of the spacecraft shell, the bearing frame, the metal sheet and the switch in the packaging layer, and the structural material of the rocket engine. Nanocrystalline materials have better mechanical properties than ordinary materials with the same chemical composition due to their small particles. Metal Ni and its alloys are the indispensable traditional metal materials in the field of aerospace, automobile and National defense military. Nanocrystalline metal has a very good engineering application prospect, owing to it can exhibit excellent lasting strength characteristics at extremely high working temperature. At present, it is difficult to prepare large size and high-performance nanocrystalline metal. In this paper, large size bulk nanocrystalline $\mathrm{Ni}$ is prepared by electrochemical deposition, and its density, hardness and microstructure are characterized. The research results have high academic value and practical significance in engineering.

\section{Preparation of nanocrystalline Ni}

\subsection{Preparation equipment}

In this paper, bulk nanocrystalline Ni was prepared by electrochemical deposition. The equipment used is as follows:

(1) Power source: SMD-30 type digitally controlled double pulse electroplating power supply is used. The maximum peak current is $10 \mathrm{~A}$, and it can provide both direct current and pulsed electrodeposition methods. 
(2) Constant temperature water bath box: control bath temperature, temperature adjustable in the range of $25^{\circ} \mathrm{C}$ to $100{ }^{\circ} \mathrm{C}$, the temperature of this experiment is $50{ }^{\circ} \mathrm{C}$;

(3) Electroplating bath: the electrodeposition plating bath is made of PVP plastic. It is characterized by heat resistance, no deformation at $50^{\circ} \mathrm{C}$, and good insulation.

(4) Cathode and anode hangers;

(5) Agitator: Electro-deposition selects self-control motor agitator.

(6) $\mathrm{pH}$ measurement: PHS-25B is used to measure the $\mathrm{pH}$ value of electrodepositing effusion.

The diagram of the direct current electrodeposition equipment is shown in Figure 1.

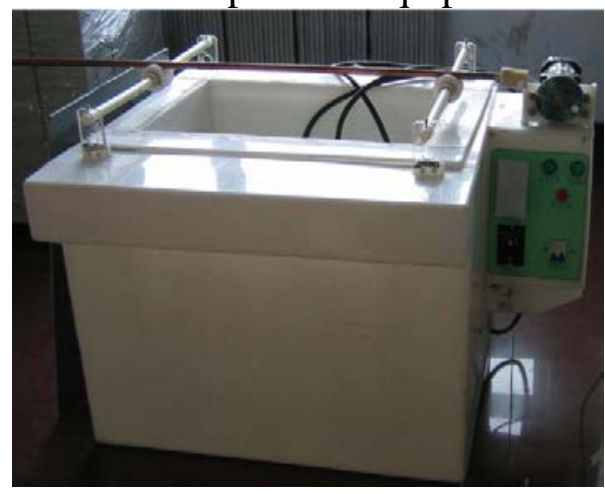

Fig.1 The device for depositing nanocrystalline $\mathrm{Ni}$

\subsection{Preparation of ingredient}

The ingredients required for the preparation process include anode plate, cathode plate and electrolyte. The specific ingredients are as follows:

(1) Anode plate: electrolytic nickel plate (purity 99.96\%) with size $18.0 \mathrm{~cm} \times 10.0 \mathrm{~cm} \times 1.0 \mathrm{~cm}$ (long, wide, thick);

(2) Cathode plate: stainless steel plate with size $15.0 \mathrm{~cm} \times 10.0 \mathrm{~cm} \times 0.1 \mathrm{~cm}$ (long, wide and thick)

(3) The chemical reagent required for the electrolyte: the main salt of the solution (nickel sulfate, nickel chloride, boric acid), surfactant (saccharin), $\mathrm{pH}$ value regulator (sulfuric acid and sodium hydroxide), all the chemical reagents are analytically pure, as shown in Table 1.

Table 1 Electrolyte composition of electrodeposited nanocrystalline $\mathrm{Ni}$

\begin{tabular}{ccc}
\hline $\begin{array}{c}\text { Composition of } \\
\text { electrodepositing fluid }\end{array}$ & Chemical formula & Content (g/l) \\
\hline Nickel sulfate & $\mathrm{Ni}\left(\mathrm{SO}_{3} \mathrm{NH}_{2}\right)_{2} \cdot 4 \mathrm{H}_{2} \mathrm{O}$ & 300 \\
Nickel chloride & $\mathrm{NiCl}_{2} \cdot 6 \mathrm{H}_{2} \mathrm{O}$ & 15 \\
Boric acid & $\mathrm{HBO}_{3}$ & 30 \\
Saccharin & $\mathrm{C}_{7} \mathrm{H}_{5} \mathrm{NO}_{3} \mathrm{~S}$ & $0.1 \sim 1$ \\
Distilled water & $\mathrm{H}_{2} \mathrm{O}$ & \\
\hline
\end{tabular}

\subsection{Electrochemical deposition process}

\subsubsection{Cathode and Anode Plate Treatment:}

The Ni board was coated with polypropylene cloth to electrolyse the sediment to prevent the sediment generated during the anodic dissolution from entering the electrolyte. The anode electrolysis Ni plate and the cathode stainless steel plate are polished in order of 600\#, 1000\#, 1500\# and 2000\# SiC sand paper, remove the surface pollutants and oxides, put them in acetone, clean the degreasing $5 \mathrm{~min}$, rinse with ethanol, and clean them with deionized water at last. The anode electrolysis Ni plate of the dryer is used, and the cathode stainless steel plate is soaked in 5\% dilute hydrochloric acid solution and soaked in the activated $2 \mathrm{~min}$ to provide the highly active surface for the electrode reaction. 


\subsubsection{Electrolyte configuration:}

The nickel chloride (15g/l), nickel sulfate $(280 \mathrm{~g} / \mathrm{l})$ and boric acid $(35 \mathrm{~g} / \mathrm{l})$ were respectively weighed by FA1004 type electronic analytical balance. The boric acid is heated to 80 degrees centigrade to dissolve quickly. After the solvent was dissolved evenly, the $\mathrm{pH}$ value of the solution was less than 3, and 5 10\% diluted $\mathrm{NaOH}$ solution was used to titrate until the $\mathrm{pH}$ value was 4.5 5.0.

\subsubsection{Hull cell experiment:}

In the electroplating process, adding a small amount of surfactant can improve the quality of the coating. Saccharin $\left(\mathrm{C}_{7} \mathrm{H}_{5} \mathrm{NO}_{3} \mathrm{~S}\right)$ can refine grains and reduce the tensile stress in the coating. It is very important to choose suitable additives, owing to the experiment show that excessive surfactants can cause the surface of the coating to be unclean or even embrittlement. Hull cell experiment was also carried out in order to obtain suitable additive content. The copper foil with thickness of $2 \mathrm{~mm}$ was plated at different current densities for 10 minutes. The suitable additive content and the optimal current density region were obtained by surface observation and bending test. It is found that the critical concentration of $\mathrm{C}_{7} \mathrm{H}_{5} \mathrm{NO}_{3} \mathrm{~S}$ is $1.2 \mathrm{~g} / \mathrm{l}$, and the plating is embrittlement when the concentration is too high. The current density range obtained from Hull cell experiment is $1 \mathrm{~A} / \mathrm{dm}^{2}$ to $8 \mathrm{~A} / \mathrm{dm}^{2}$, and in this range, the brightness of the coating increases with the increase of current density.

\subsubsection{Prophylaxis of marginal stomatal and accumulated burl:}

In the process of plating, it is easy to produce the marginal stomatal and accumulated burl, on account of the current density is inhomogeneous at the edge of the cathode plate, and the current density at the edge is high. In order to prevent the generation of edge stomata and tumor, PVP plastic is used as a rectangular mold, and the width of the frame is about $2 \mathrm{~cm}$ wider than the that of cathode plate. The groove is processed inside the frame, and the groove width is slightly larger than the thickness of the cathode plate. In the process of plating, the cathode stainless steel plate is placed in the rectangular mold to embed the groove, avoiding the high current density of the edge, preventing the formation of the marginal stomatal and accumulated burl.

\subsubsection{Temperature and $\mathrm{pH}$ control of plating process:}

The temperature of the liquid is $50^{\circ} \mathrm{C}$, stirring continuously through a blender, improving the uniformity of the solution, reducing the concentration polarization, and effectively removing bubbles. The $\mathrm{pH}$ value of the plating bath is between 4.5 5.0, and the $\mathrm{pH}$ value of the solution is measured every hour after one hour and adjusted by dilute sulfuric acid. The XRD characterization of nanocrystalline Ni prepared by different current density by adjusting the current density is shown in Figure 2. The relation between grain size and current density calculated by XRD is shown in Table 2. According to the surface state and grain size of the sample, the optimum parameters of nanocrystalline $\mathrm{Ni}$ by direct current electrodeposition are as follows: current density is $7 \mathrm{~A} / \mathrm{dm}^{2}, \mathrm{pH}$ value is between $4.5 \sim 5.0, \mathrm{C}_{7} \mathrm{H}_{5} \mathrm{NO}_{3} \mathrm{~S}$ content is $1 \mathrm{~g} / \mathrm{l}$, the temperature of liquid is $50{ }^{\circ} \mathrm{C}$.

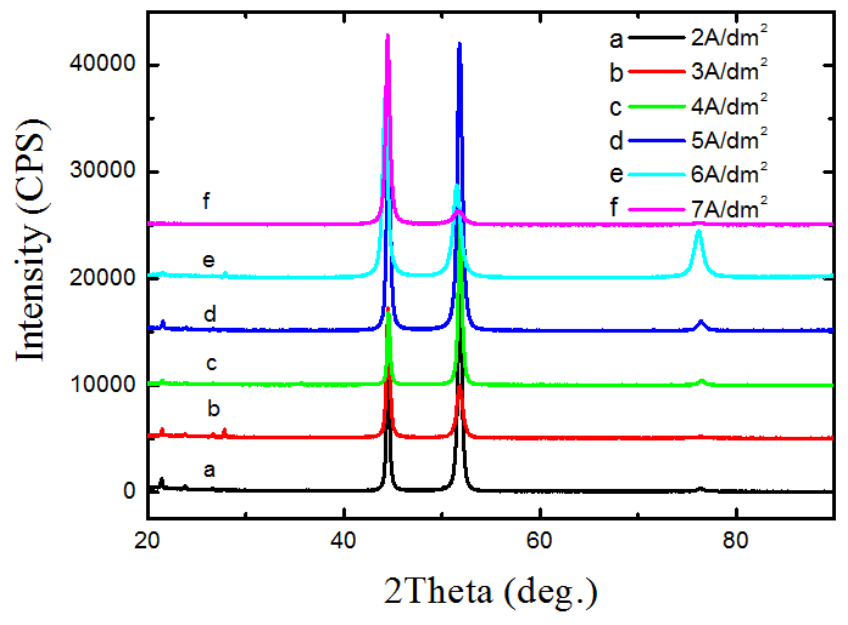

Fig.2 The XRD results of different current density 
Table2 The average grain size of the different current density electrodeposition

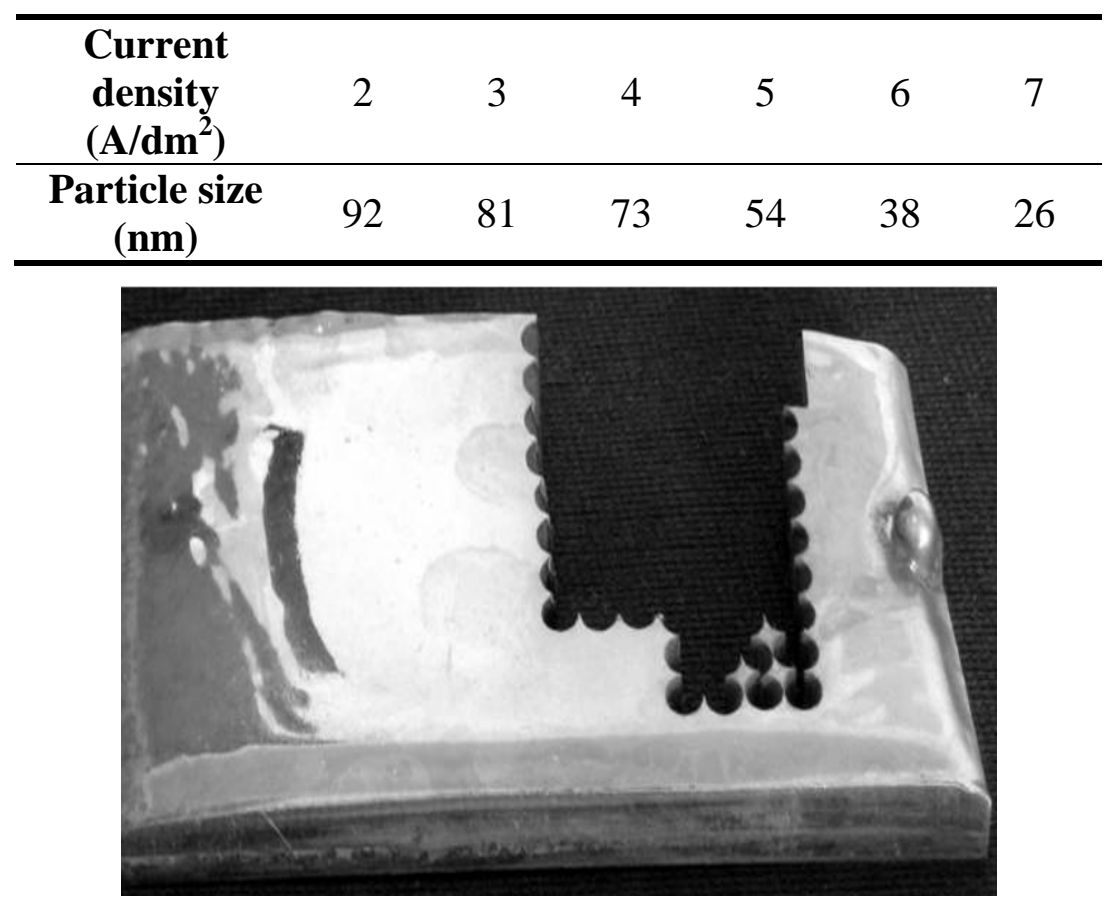

Fig.3 Direct-current electrodeposition method bulk nanocrystalline Ni plating

\section{Characterization of nanocrystalline $\mathrm{Ni}$}

\subsection{Density measurement}

The sample is measured by the true density method. The Archimedes's Law ( $\mathrm{PV}=\mathrm{nRT}$ ) of inert gas under certain conditions is obtained through the Archimedes gas expansion replacement method. The actual volume of the sample is accurately measured by the reduction of the gas volume in the test chamber by placing the sample into the test chamber, and the true density is the mass / real volume. True density measurement is completed in Beijing Beesd Instrument Technology (Beijing) Co., Ltd., the gas is high purity nitrogen of purity $>99.999 \%$, the product model is 3H-2000TD1 fully automated true density analyzer, the accuracy is better than $\pm 0.04 \%$, repeatability is better than $\pm 0.02 \%$, and the resolution is $0.0001 \mathrm{~g} / \mathrm{cm}^{3}$. The density of Electrodeposited Nanocrystalline $\mathrm{Ni}$ is $8.8343 \mathrm{~g} / \mathrm{cm}^{3}$.

\subsection{Hardness measurement}

The surface hardness of nanocrystalline Ni and coarse grained Ni was measured by HVS-1000 digital microhardness tester. The digital microhardness tester is mainly composed of the loading system, the optical system and the special microprocessor. Through the optical amplification, the length of the indentation diagonal line is measured on the surface of the diamond body under the load under a certain load, and the Vickers hardness is calculated by the length value. The calculation method is as follows:

$$
\begin{aligned}
& H v=\frac{2 P \sin (\alpha / 2)}{d^{2}} \square \square \square \square \\
& d=N / V
\end{aligned}
$$

In Equation (1), the $\alpha$ is the angle between two faces of the square four pyramid $\left(\alpha=136^{\circ}\right)$; $P$ is the load; $D$ is the diagonal length of the indentation; $N$ is the length of the indentation diagonal on the micro eyepiece; $V$ is the magnification of the objective lens. Before the measurement, the surface of the coating is polished. Take 5 different positions on each sample surface for testing, and take the 
average value as the measurement result. The load was $1 \mathrm{~kg}$ and the loading time was $20 \mathrm{~s}$. The average hardness of the nanocrystalline $\mathrm{Ni}$ was $384.1 \mathrm{HV}_{1.0}$.

\subsection{Microstructure characterization}

Transmission electron microscopy (TEM, H-800, Japan) was used to observe the microstructure of nanocrystalline $\mathrm{Ni}$ and conventional coarse-grained $\mathrm{Ni}$, and the sample was subjected to Selected area electron diffraction (SAD) analysis. Figure 4 Transmission Electron Micrograph of Nanocrystalline Ni Prepared by Electrodeposition, and the illustration is an electoral electron diffraction pattern.

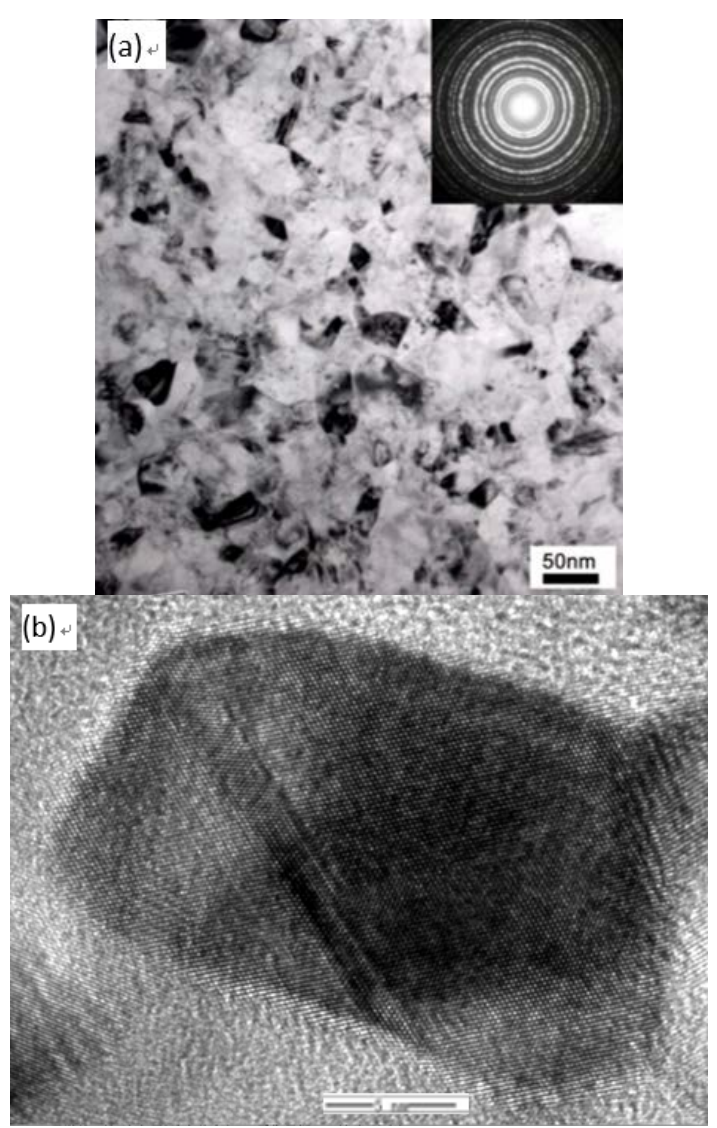

Fig.4 TEM micrographs showing microstructure of (a) nanocrystalline Ni and (b) conventional Ni

The TEM photographs of nanocrystalline Ni deposited by multiple direct currents are used to count multiple grains, and the grain size distribution is shown in Fig. 5. The particle size distribution is narrow, and the average grain size is $26 \mathrm{~nm}$ according to the number of statistics, which is in agreement with the XRD results. It shows that XRD is more accurate to evaluate the average grain size of nanocrystalline materials.

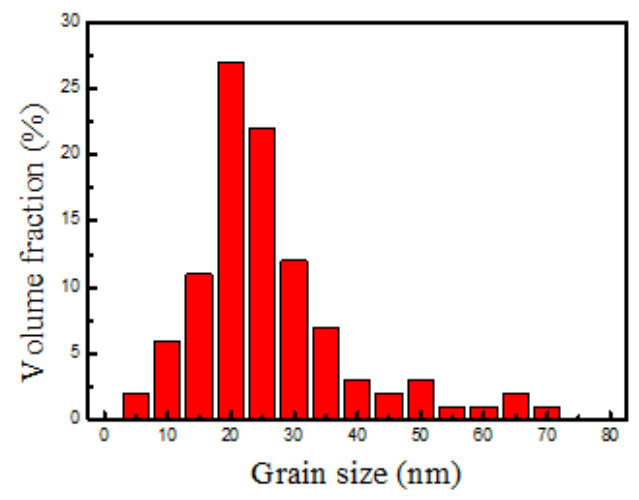

Fig.5 Grain size distribution 


\section{Conclusion}

High quality nanocrystalline Ni was prepared by electrochemical deposition, by purifying the plating bath, adjusting the current density and adjusting the content of additives reasonably. The experiments show that the three-dimensional size of nanocrystalline $\mathrm{Ni}$ prepared by electrodeposition is more than $5 \mathrm{~mm}$, the particle size is uniform, the grain size is $26 \mathrm{~nm}$, the density is $8.8343 \mathrm{~g} / \mathrm{cm} 3$, the average hardness is $384.1 \mathrm{HV}_{1.0}$.

\section{References}

[1] Embury J, Fisher R. The structure and properties of drawn pearlite. Acta Metallurgica, 1966, 14(2): 147-159.

[2] Armstrong $\dagger$ R, Chou Y, Fisher R, Louat N. The limiting grain size dependence of the strength of a polycrystalline aggregate. Philosophical Magazine, 1966, 14(131): 943-951.

[3] Gleiter H. Nanostructured materials. Advanced Materials, 1992, 4(7-8): 474-481.

[4] Haubold T, Birringer R, Lengeler B, Gleiter H. EXAFS studies of nanocrystalline materials exhibiting a new solid state structure with randomly arranged atoms. Physics Letters A, 1989, 135(8): 461-466.

[5] Henglein A. Small-particle research: physicochemical properties of extremely small colloidal metal and semiconductor particles. Chemical Reviews, 1989, 89(8): 1861-1873.

[6] Klabunde K J, Stark J, Koper O, Mohs C, Park D G, Decker S, Jiang Y, Lagadic I, Zhang D. Nanocrystals as stoichiometric reagents with unique surface chemistry. The Journal of Physical Chemistry, 1996, 100(30): 12142-12153.

[7] Yiping L, Hadjipanayis G, Sorensen C, Klabunde K. Magnetic properties of fine cobalt particles prepared by metal atom reduction. Journal of Applied Physics, 1990, 67(9): 4502-4504.

[8] Awschalom D, McCord M, Grinstein G. Observation of macroscopic spin phenomena in nanometer-scale magnets. Physical review letters, 1990, 65(6): 783-786.

[9] Gleiter H. Nanostructured materials: State of the art and perspectives. Nanostructured materials, 1995, 6(1): 3-14.

[10] Gleiter H. Nanostructured materials: basic concepts and microstructure. Acta materialia, 2000, 48(1): 1-29. 